\title{
Two Multiple Sclerosis Relapses Affecting the Left Pontine-Mesencephalic Transition and Later the Right Mid Pons, With Distinct Eye Movement Abnormalities - The Importance Of Semiology Above Medical Imaging: Case Report
}

\author{
Authors: $\quad$ *Paulo Afonso Mei, $\quad$ ㄱ, Francisco Saulo S.C. Tavares ${ }^{1}$ \\ 1. University of Campinas (UNICAMP), São Paulo, Brazil \\ 2. São Leopoldo Mandic College, Campinas, Brazil \\ *Correspondence to drkult@gmail.com \\ Disclosure: The authors have declared no conflict of interest. \\ Acknowledgements: The patient gave full consent for this publication. \\ Received: $\quad 29.12 .19$ \\ Accepted: $\quad 29.01 .20$ \\ Keywords: Internuclear ophthalmoplegia, multiple sclerosis (MS), normal appearing white matter \\ (NAWM), semiology, throclear nerve palsy. \\ Citation: \\ EMJ Radiol. 2020;1[1]:89-93.
}

\begin{abstract}
The authors report the case of a 22-year-old female patient who presented with two distinct relapses of multiple sclerosis. The first was a lesion in the dorsal paramedian pontine area to the left, and the second, not visible in the MRI but presumably affecting the right medial longitudinal fasciculus, in the pons. In both cases, the semiology was unquestionable and compatible to the proposed areas of demyelination, with the patient fully recovering her eye movements after pulses of methylprednisolone.
\end{abstract}

\section{INTRODUCTION}

Multiple sclerosis (MS) is a demyelinating disorder, in which both humoral and cellular mechanisms of autoimmunity coexist, ${ }^{1,2}$ affecting 15 of every 100,000 Brazilians ${ }^{3}$ and between $<1$ and 38 per 100,000 in other regions of Latin America. ${ }^{4}$ The majority of patients are white females $>30$ years of age. ${ }^{5}$ Despite constant advancements in research and the development of new medications ${ }^{6}$ that have to some extent prolonged survival rate, ${ }^{7}$ mortality in the MS population is still around three times higher than the corresponding general population. ${ }^{8,9}$
Cerebellar and/or brainstem lesions in MS happen roughly in one third of the relapses. ${ }^{10}$ The authors report a case of two relapses that occurred in a female patient, compatible with demyelination of different areas in the brainstem and displaying a different semiological finding during each relapse.

\section{CASE REPORT}

The patient was a 22-year-old woman of mixed black and Brazilian-indigenous background who presented to the emergency department with a complaint of diplopia, numbness in the 
face, and trouble with her balance. During physical examination, she had normal strength (Grade 5) in the face and the four limbs. Cranial nerve analysis showed divergent deviation of the left eye (LE) in the neutral gaze position associated with left hypertropia, a horizontal misalignment of the eyes. Voluntary eye movement tests (Figure 1, Situation 1) displayed a deficit in the adduction (paralysis of the medial rectus) of the LE, with augmentation of hypertropia during dextroversion and limitation of lowering of the same eye during infraversion.
Although not doing so unprompted, she reported improvement of her double vision when asked to tilt her head to the right. She did not show ptosis or pupillary changes. Red glass, double Maddox rod, or prisms tests were not performed. Although the presence of paralysis of more than one eye muscle lessens the accuracy of the ParksBielschowsky three step test," this was interpreted as a possible left trochlear nerve palsy, in addition to left internuclear ophthalmoplegia.

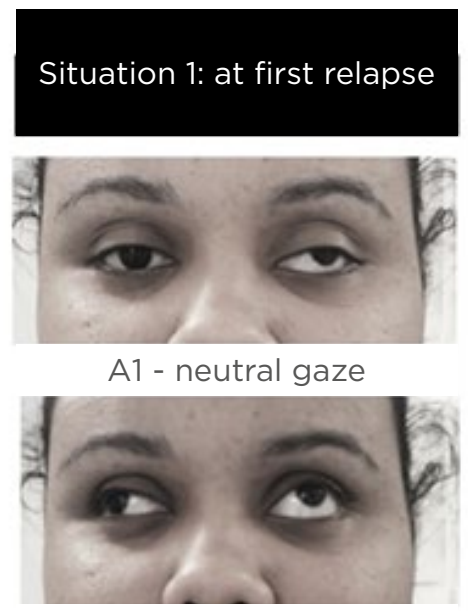

B1 - right gaze

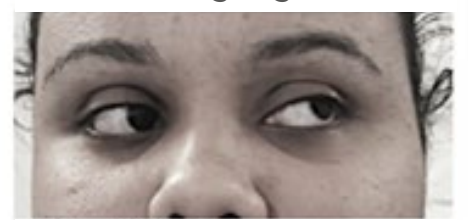

C1 - left gaze

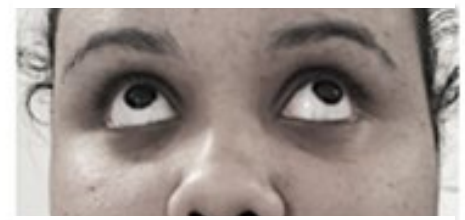

D1 - upwards gaze

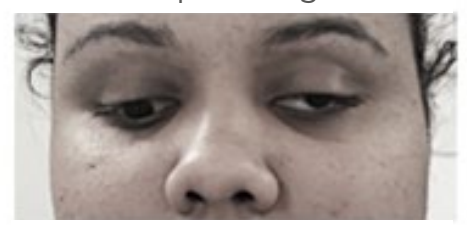

E1 - downwards gaze

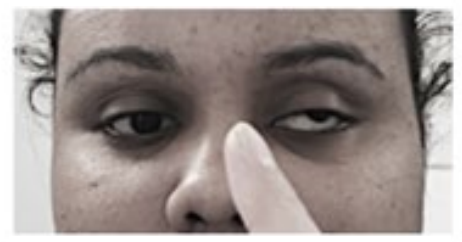

F1 - convergence

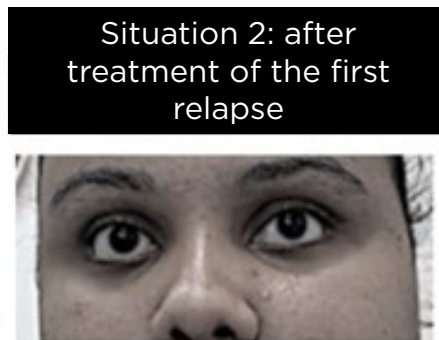

A2 - neutral gaze

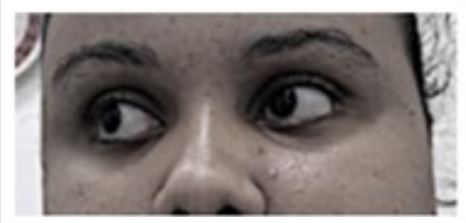

B2 - right gaze

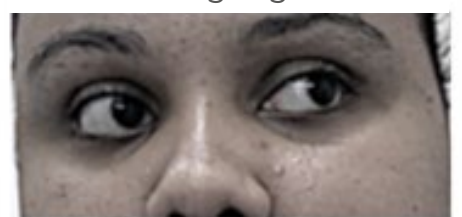

C2 - left gaze

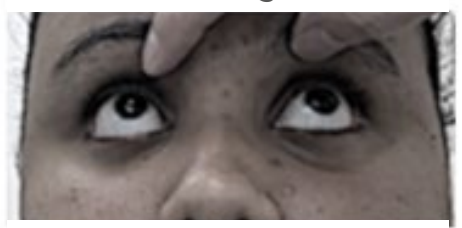

D2 - upwards gaze

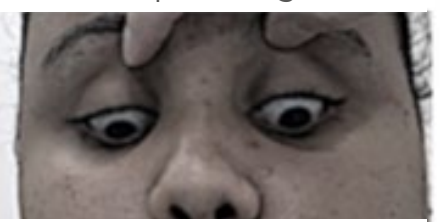

E2 - downwards gaze

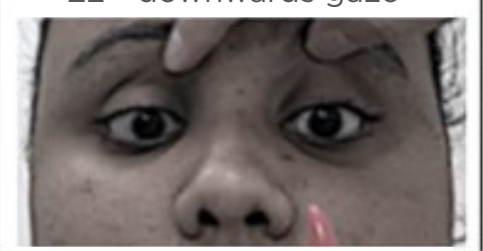

F2 - convergence

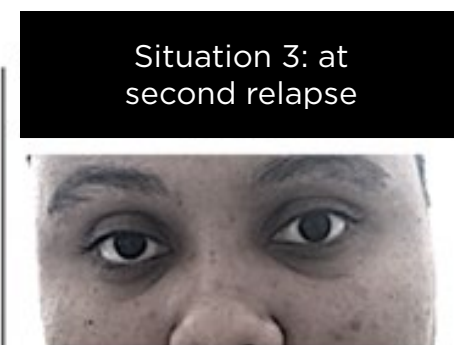

A3 - neutral gaze

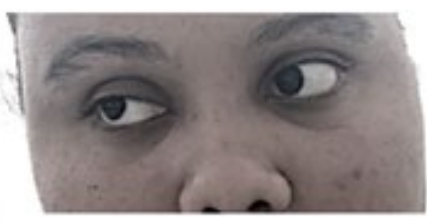

B3 - right gaze

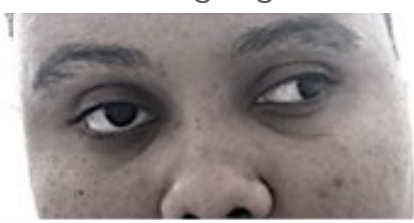

C3 - left gaze

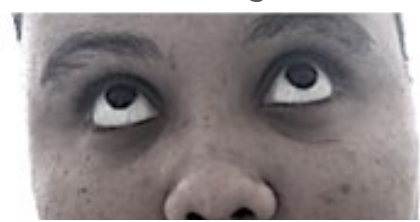

D3 - upwards gaze

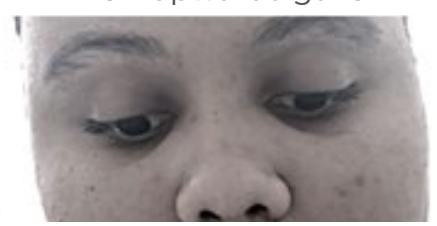

E3 - downwards gaze

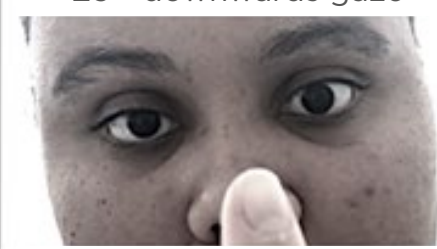

F3 - convergence

Figure 1: Examination of the eye movements of the same patient for three different situations. Situation 1: at first relapse; Situation 2: after treatment of the first relapse; and Situation 3: at second relapse. 


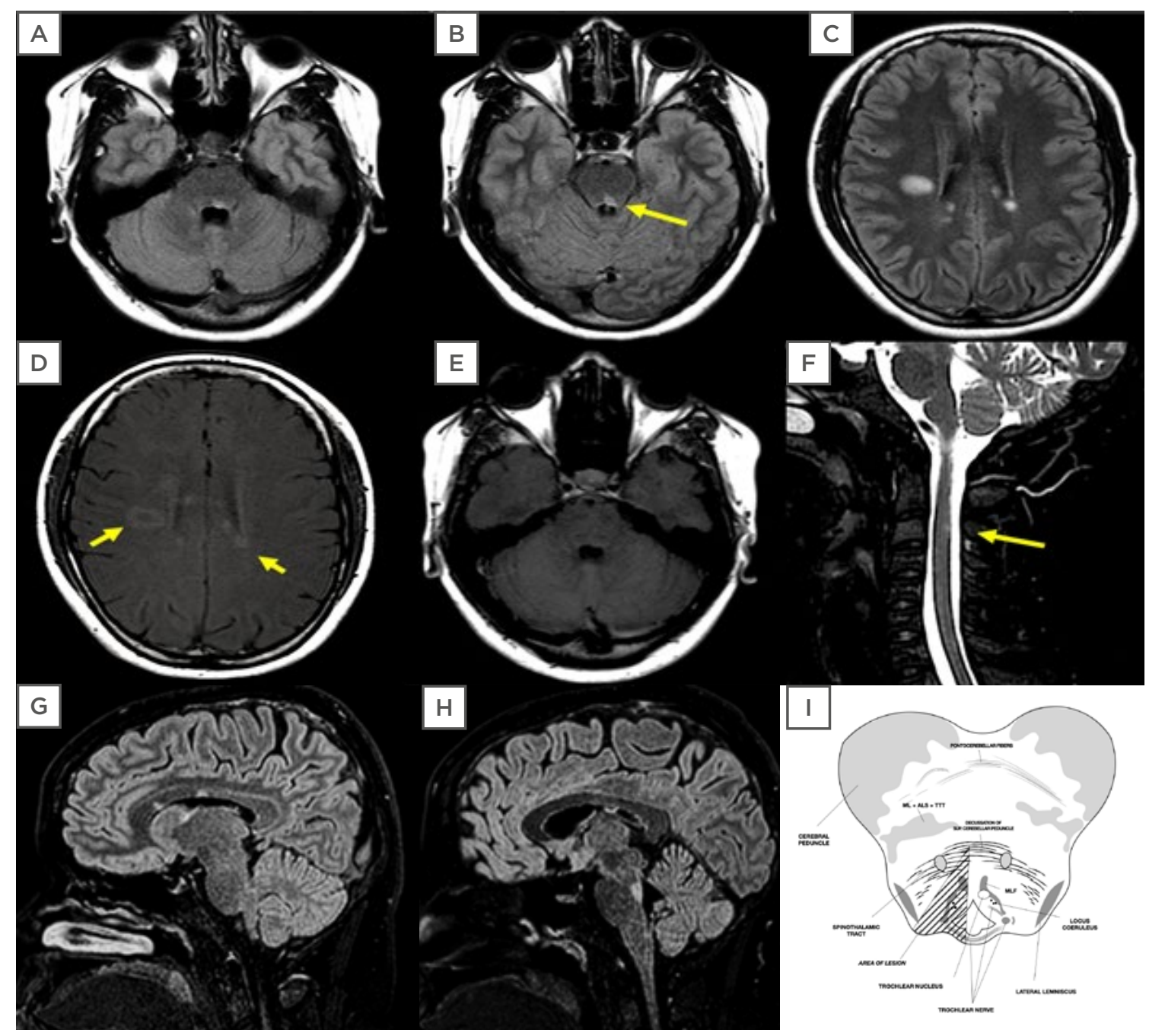

Figure 2: MRI performed at the occasion of the first relapse.

Displayed here are fluid-attenuated inversion recovery sequences (FLAIR) (A, B, C, G, H), T1 with gadolinium (D, E) and T2 acquisitions (F). Supratentorial lesions are visible (C, D, G, H), along with moderate contrast enhancement (D: arrows), which did not happen with infratentorial lesions ( $A, B, E, G, H$ ) or in the cervical C3 lesion (F: arrow). (I) anatomical representation of the affected area (hatched triangle) of the midbrain pontine transition that is displayed in the arrow in B. The images fulfil radiological criteria for dissemination in time and space, as required in multiple sclerosis.

ALS: anterolateral system; ML: medial lemniscus; MLF: medial longitudinal fasciculus; TTT: trigeminal thalamic tract. Illustration (I): Adapted from University of Wisconsin-Madison.13

Laboratory exams excluded renal, hepatic, rheumatic, or endocrine abnormalities, as well as confirmed negative serology for hepatitis $B$ and C, syphilis, and HIV. Serum levels of vitamin B12 and vitamin $\mathrm{D}$ were both within normal range (426 pg/mL and $41 \mathrm{pg} / \mathrm{mL}$, respectively). White blood cell count displayed discrete leukocytosis $\left(12.3 \times 10^{3}\right.$ cells $/ \mathrm{mm}^{3}$ [92\% segmented cells]), and C-reactive protein levels of $17 \mathrm{mg} / \mathrm{L}$ (normal: $<10$ $\mathrm{mg} / \mathrm{L}$ ), which was attributed to a sinus infection since she also presented with facial pain and purulent nasal discharge. Lumbar puncture revealed 1 leukocyte $/ \mathrm{mm}^{3}$ and protein levels of 22 $\mathrm{mg} / \mathrm{dL}$, both within normal value ranges. ${ }^{12}$

MRI of the whole neural axis was performed (Figure 2), showing multiple oval-shaped, hyperintense lesions in T2 and fluid-attenuated inversion recovery sequences in the supratentorial subcortical and periventricular white matter. Some exhibited a perivenular distribution pattern, and others alongside the callosal-septal interface, with contrast enhancement in at least three periventricular lesions and in one lesion 


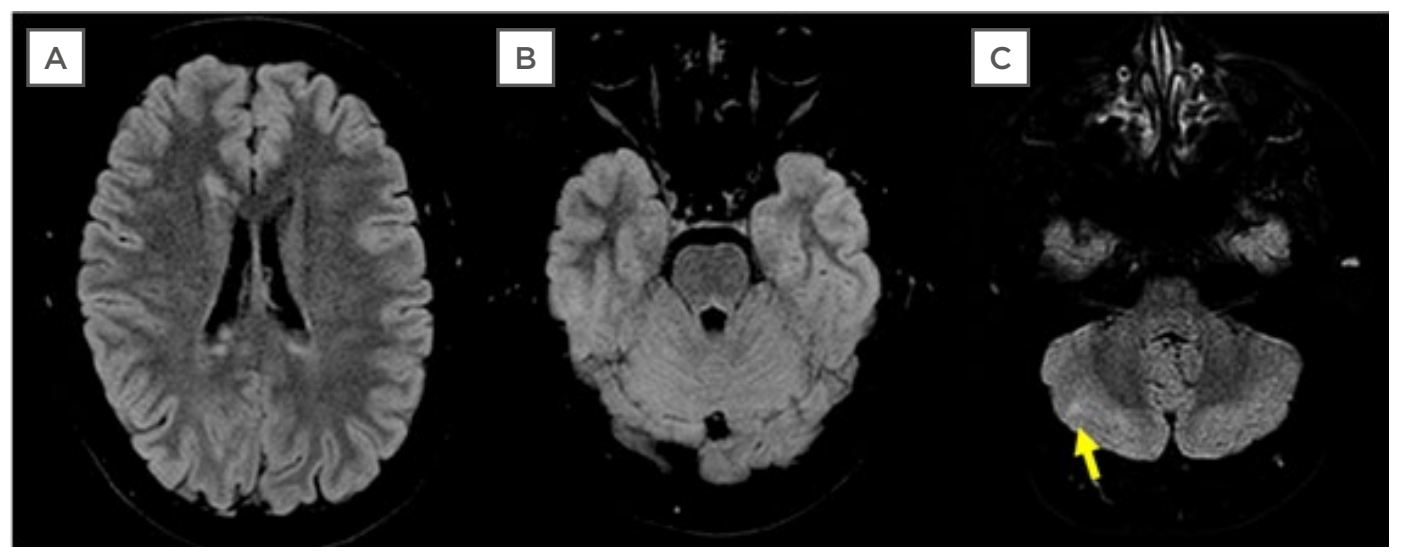

Figure 3: MRI (FLAIR Acquisition) performed by the occasion of the second relapse, 2 months after the first. Although one can see reduction of the prior lesions in $A$ and $B$, and that no new viewable lesions at the image could explain the clinical exam, which pointed to damage to the right MLF, there is at least one viewable lesion at the cerebellar right hemisphere, indicating that the disease at the moment remained active.

MLF: medial longitudinal fasciculus.

at the right centrum semiovale. There was also another hyperintense, nonenhanced lesion at the posterior medial part of the left pons, extending up to the level of the facial colliculus, which probably resulted in damage to the left trochlear nucleus and the medial longitudinal fasciculus, explaining the findings of the clinical examination. However, by the posterior cervical spine the MRI exposed a hyperintense T2 lesion at C3 level. These aspects fulfilled radiologic criteria for dissemination in space and time of MS. ${ }^{14}$

The patient underwent pulse therapy with intravenous methylprednisolone, the standard treatment for MS relapses, ${ }^{15} 1 \mathrm{~g}$ once daily for 5 days plus antibiotics for the sinus infection, and showed an improvement of ocular movements at discharge, however the complaint of gait impairment was maintained and the patient required a walking cane. One month later, examination in the consultation room revealed normal ocular movements (Figure 1, Situation 2).

Two months after the first admission, the patient developed a new complaint of diplopia. This time, during physical examination, she had a normal range of extrinsic movements of the LE and deficit in the adduction (medial rectum paresis) of the right eye. This suggested a new and different lesion, topographically compatible with a lesion of the right medial longitudinal fasciculus (Figure 1, Situation 3). This time, no hypertropia was present and the eyes were horizontally aligned. Once again, she did not display ptosis nor pupillary changes and this examination was interpreted as a probable right internuclear ophthalmoplegia. A follow-up MRI performed 3 days after the new symptoms onset (and prior to new steroid course) (Figure 3) did not reveal any new lesions in the brainstem, nor any gadolinium enhancement, however there was a small, new lesion at the right cerebellum, suggesting maintenance of the disease activity. Despite the lack of visualisation of the lesion in the expected area, this was interpreted as a new relapse affecting the right pontine area. This was contralateral to the first relapse because the eye movement abnormalities could not be faked, as might be possible in a conversive disorder, therefore demonstrating organicity. Following this, the patient was put on a new regimen of intravenous methylprednisolone (1 $\mathrm{g}$ once daily for 5 days) resulting in full ocular movement normalisation but retention of ataxic gait and the occasional use of a walking cane.

Currently, the patient is still being followed up with intravenous use of natalizumab (anti a-4integrin monoclonal antibody), ${ }^{16}$ and presents partial remission of symptoms in the inferior limbs and no further relapses to the date of this report. 


\section{DISCUSSION}

The authors consider this case worthy of scientific highlight for two main reasons: first, because of the extensiveness of the physical exam involved, which exposed the involvement of two very different regions (and different sides) of the brainstem, causing distinct semiological findings. This is of note because the involvement of the trochlear nucleus is rare (most of the causes of IV nerve palsy are usually of lesions of the cranial nerve itself). ${ }^{17,18}$ The second reason is to emphasise the so called clinicoradiological paradox, a fundamental challenge where new relapses in a MS patient with an organic substrate might comprise very small areas, to the point of not being recognised as new lesions in the MRI. Despite the use of gadolinium, ${ }^{19}$ there is always a chance of disease activity in the normal appearing white matter. ${ }^{20}$

\section{CONCLUSION}

The authors reckon that in MS cases where semiology unquestionably denotes brainstem lesion, the decision for acute treatment should be based not only upon radiological confirmation, but mainly on the clinical manifestations. This is pivotal because truncal lesions have the potential to cause permanent and considerable sequelae and are independent indicators of worse prognosis if not promptly treated. ${ }^{21}$

\section{References}

1. Korn T. Pathophysiology of multiple sclerosis. J Neurol. 2008;255(Suppl 6):2-6.

2. Dendrou CA et al. Immunopathology of multiple sclerosis. Nat Rev Immunol. 2015;15:545-58.

3. Associação Brasileira de Esclerose Múltipla (ABEM). 2020. Available at: www.abem.org.br. Last accessed: 31 January 2020

4. Cristiano E, Rojas JI. Multiple sclerosis epidemiology in Latin America: an updated survey. Mult Scler J Exp Transl Clin. 2017:3(2):2055217317715050.

5. Cardoso $\mathrm{E}$ et al. Clinical and epidemiological profile of multiple sclerosis in a reference center in the State of Bahia, Brazil. Arq Neuropsiquiatr. 2006;64(3b):727-30.

6. Torkildsen $\varnothing$ et al. Disease-modifying treatments for multiple sclerosis - a review of approved medications. Eur J Neurol. 2016;23(Suppl 1):18-27.

7. Goodin DS et al. Survival in MS: a randomized cohort study 21 years after the start of the pivotal IFNB-1b trial. Neurology. 2012;78(17):1315-22.

8. Lunde HMB et al. Survival and cause of death in multiple sclerosis: a 60year longitudinal population study.
J Neurol Neurosurg Psychiatry. 2017;88(8):621-5

9. Kaufman DW et al. Survival in commercially insured multiple sclerosis patients and comparator subjects in the U.S. Mult Scler Relat Disord. 2014;3(3):364-71.

10. Negreiros AA et al. Clinical and epidemiological profile of patients diagnosed with multiple sclerosis in João Pessoa, Paraíba, Brazil. Arq Neuropsiquiatr. 2015;73(9):741-5.

11. American Academy of Ophthalmology EyeWiki. Three step test for cyclovertical muscle palsy. 2019. Available at: https://eyewiki. aao.org/Three_Step_Test_for Cyclovertical_Muscle_Palsy. Last accessed: 31 January 2020.

12. MSD Manual Professional Version. CSF tests: normal values. 2018 Available at: https://www. msdmanuals.com/professional/ resources/normal-laboratoryvalues/csf-tests-normal-values. Last accessed: 31 January 2020.

13. University of Wisconsin-Madison. Brain Stem. Available at: http://www. neuroanatomy.wisc.edu/coursebook/ webstem.pdf. Last accessed: 31 January 2020.
14. Thompson AJ et al. Diagnosis of multiple sclerosis: 2017 revisions of the McDonald criteria. Lancet Neurol. 2018;17(2):162-73.

15. Goodin DS. Glucocorticoid treatment of multiple sclerosis. Handb Clin Neurol. 2014;122:455-64

16. Hutchinson M. Natalizumab: a new treatment for relapsing remitting multiple sclerosis. Ther Clin Risk Manag. 2007;3(2):259-68.

17. Tiffin PA. Acquired palsy of the oculomotor, trochlear and abducens nerves. Eye (Lond). 1996;10(Pt 3):37784

18. Mansour AM, Reinecke RD. Central trochlear palsy. Survey of Ophthalmology. 1986;30(5):279-8.

19. Maniega SM et al. White matter hyperintensities and normalappearing white matter integrity in the aging brain. Neurobiol Aging. 2015;36(2):909-18

20. Mews I et al. Oligodendrocyte and axon pathology in clinically silent multiple sclerosis lesions. Mult Scler. 1998;4(2):55-62.

21. Damasceno A et al. Prognostic indicators for long-term disability in multiple sclerosis patients. J Neurol Sci. 2013;324(1-2):29-33. 